\title{
Spontaneous symmetry breaking in pure 2D Yang-Mills theory
}

\author{
G. Aminov®* \\ Department of Physics and Astronomy, Stony Brook University, Stony Brook, New York 11794, USA \\ and ITEP NRC KI, Moscow 117218, Russia
}

(Received 10 February 2020; accepted 11 May 2020; published 26 May 2020)

\begin{abstract}
We consider purely topological 2D Yang-Mills theory on a torus with the second Stiefel-Whitney class added to the Lagrangian in the form of a $\theta$ term. It will be shown that at $\theta=\pi$ there exists a class of $S U(2 N) / \mathbb{Z}_{2}(N>1)$ gauge theories with a twofold degenerate vacuum, which spontaneously breaks the time reversal and charge conjugation symmetries. The corresponding order parameter is given by the generator $\mathcal{O}$ of the $\mathbb{Z}_{N}$ one-form symmetry.
\end{abstract}

DOI: 10.1103/PhysRevD.101.105017

\section{INTRODUCTION}

The possibility of having a number of degenerate vacua called $\theta$-vacua in two dimensional gauge theories was studied in the 1970s by a number of authors [1-6]. Both Abelian and non-Abelian theories were considered, and the existence of the multiple vacua was shown to be independent of the spontaneous symmetry breaking of the gauge symmetry. Instead, the presence of some matter fields, either fermionic or scalar, was required.

In this paper we consider purely topological 2D YangMills theory on a torus with the second Stiefel-Whitney class added to the Lagrangian in the form of a $\theta$ term. It will be shown that at $\theta=\pi$ there exists a class of $S U(2 N) / \mathbb{Z}_{2}$ $(N>1)$ gauge theories with a twofold degenerate vacuum. These two vacuum states are related by the time reversal or the charge conjugation and thus indicate the spontaneous symmetry breaking. The corresponding order parameter is given by the generator $\mathcal{O}$ of the $\mathbb{Z}_{N}$ one-form symmetry with the following action of the charge conjugation on it:

$$
\mathrm{COC}^{-1}=\mathcal{O}^{-1}
$$

The motivation to consider such theories comes from the recent developments in generalized global symmetries and 't Hooft anomalies [7-27]. In particular, authors of [16] considered $S U(N)$ gauge theory in four dimensions and showed that at $\theta=\pi$ there is the discrete 't Hooft anomaly involving time reversal and the center symmetry. As a consequence of this anomaly, the vacuum at $\theta=\pi$ cannot

\footnotetext{
'gleb.aminov@stonybrook.edu
}

Published by the American Physical Society under the terms of the Creative Commons Attribution 4.0 International license. Further distribution of this work must maintain attribution to the author(s) and the published article's title, journal citation, and DOI. Funded by SCOAP . be a trivial nondegenerate gapped state. Another example of the 't Hooft anomaly constraining the vacuum of the theory is related to the $2 \mathrm{D} \mathbb{C P}^{n-1}$ model [15], where for $n>2$ the mixed anomaly between time reversal symmetry and the global $\operatorname{PSU}(n)$ symmetry at $\theta=\pi$ leads to the spontaneous breaking of time reversal symmetry with a twofold degeneracy of the vacuum [28]. The list of examples could be longer, but we will conclude by mentioning the works $[12,13]$, where the 't Hooft anomalies for discrete global symmetries in bosonic theories were studied in two, three, and four dimensions. Although, in this paper, we are not going to discuss the possible relation of the spontaneous symmetry breaking to the anomaly, one could hypothesize the existence of the mixed anomaly between $(-1)$-form symmetry and the charge conjugation in the theories under consideration. ${ }^{1}$

Recently, we became aware of the paper by Kapec, Mahajan, and Stanford [25], which has partial overlap with our results for the partition functions of $P S U(N)$ gauge theories. In [25] the higher genus partition functions were computed and utilized in the context of random matrix ensembles. As we will show in the main text of the paper, there is no spontaneous symmetry breaking for the case of $P S U(N)$ gauge theories, and thus, the main results of our study are not covered in [25]. Also, the paper by Sharpe [29] discussing one-form symmetries in the various 2D theories appeared recently. This paper studies the connection with the cluster decomposition and is based on a number of previous results (to name a few [30-32]).

The paper is organized as follows. In Sec. II, we review the Hamiltonian approach for computing the partition functions of the pure gauge theories in two dimensions. This method originates from the work of Migdal [33] and was extensively developed in the 1980s and 1990s alongside other approaches for studying the 2D Yang-Mills

\footnotetext{
${ }^{1}$ This possibility was pointed out by Komargodski.
} 
theories [34-46]. We would also like to mention the path integral approach by Blau and Thompson [47-49], which leads to the same results but requires more involved mathematical structures. In Sec. III we use 't Hooft's twisted boundary conditions [50] to compute the partition function of the $S U(2) / \mathbb{Z}_{2}$ gauge theory. This computation is equivalent to the approach used by Witten [40] to compute the $S O(3)$ partition function starting from the $S U(2)$ gauge theory. We conclude Sec. III by introducing the $\theta$ term to the Lagrangian and computing the partition function at $\theta=\pi$, which repeats one of the results of $[51,32]$. In Sec. IV we extend all of the previous arguments to the case of $S U(N) / \mathbb{Z}_{N}$ theory. However, since there is no spontaneous symmetry breaking in $P S U(N)$ theory for any $N$, in Sec. V we focus on the more general case of $S U(N) / \Gamma$, where $\Gamma$ is the subgroup of the center of $S U(N)$. Indeed, we find out that there exists a class of $S U(2 N) / \mathbb{Z}_{2}, N>1$ theories with two vacuum states given by the fundamental and antifundamental representations of $S U(2 N)$. Additionally, we argue that there exists a broader class of $S U(2 \mathrm{Nm}) / \mathbb{Z}_{2 m}$ theories with degenerate vacuum. Finally, in Sec. VI we relate the twofold degeneracy of the vacuum to the spontaneous breaking of $\mathbf{C}$ and $\mathbf{T}$ symmetries.

\section{REVIEW: $S U(2)$ GAUGE THEORY}

To derive the answer for the partition function on the torus, we consider the canonical quantization of the theory on a cylinder (see Fig. 1). The corresponding propagator $[33,40,45]$ is given by

$$
Z\left(a, U_{1}, U_{2}\right)=\sum_{R} \chi_{R}\left(U_{1}\right) \chi_{R}\left(U_{2}\right) \mathrm{e}^{-a C_{2}(R)},
$$

where $a=e^{2} L T / 2$ is proportional to the surface area of the cylinder. The final answer for the partition function on the torus comes from gluing together the opposite sides of the cylinder:

$$
\begin{aligned}
Z & =\int \mathrm{d} U_{1} Z\left(a, U_{1}, U_{1}^{-1}\right) \\
& =\sum_{R} \mathrm{e}^{-a C_{2}(R)} \int \mathrm{d} U_{1} \chi_{R}\left(U_{1}\right) \chi_{R}\left(U_{1}^{-1}\right) .
\end{aligned}
$$

Using the identity

$$
\int \mathrm{d} U \chi_{R}(V U) \chi_{R}\left(U^{-1} W\right)=\frac{\chi_{R}(V W)}{\operatorname{dim} R},
$$

we get

$$
Z=\sum_{R} \mathrm{e}^{-a C_{2}(R)}
$$

For $S U(2)$, we have $C_{2}(R)=j(j+1)$ with half-integer $j$ and hence

$$
Z=\sum_{m=0}^{\infty} \mathrm{e}^{-a m(m+2) / 4} .
$$

\section{III. $S U(2) / \mathbb{Z}_{2}$ GAUGE THEORY}

Now we consider the cylinder as a rectangular plaquette with one pair of opposite sides being glued together. According to [50] we can introduce the following boundary conditions for the vector potential $A_{\mu}(x, t)$ :

$$
\left\{\begin{array}{l}
A_{\mu}(L, t)=\tilde{\Omega}_{1}(t) A_{\mu}(0, t), \\
A_{\mu}(x, T)=\tilde{\Omega}_{2}(x) A_{\mu}(x, 0),
\end{array}\right.
$$

with the notation $\Omega A_{\mu}=\Omega A_{\mu} \Omega^{-1}+\frac{l}{g} \Omega \partial_{\mu} \Omega^{-1}$. However, since we are using the $A_{0}=0$ gauge, we are left with time independent gauge transformations:

$$
\tilde{\Omega}_{1}(t)=\tilde{\Omega}_{1}(0) .
$$

Now making a constant gauge transformation $A_{\mu} \rightarrow \tilde{\Omega} A_{\mu}$ with

$$
\tilde{\Omega} \tilde{\Omega}_{1} \tilde{\Omega}^{-1}=\mathrm{Id}, \quad \Omega(x) \equiv \tilde{\Omega} \tilde{\Omega}_{2}(x) \tilde{\Omega}^{-1},
$$

we arrive at

$$
\left\{\begin{array}{l}
A_{1}(L, t)=A_{1}(0, t), \\
A_{1}(x, T)=\Omega(x) A_{1}(x, 0),
\end{array}\right.
$$

and the consistency condition for $\Omega$ is

$$
\Omega(0)=\Omega(L) z, \quad z \in \mathbb{Z}_{2} .
$$

FIG. 1. Holonomies on the cylinder. 


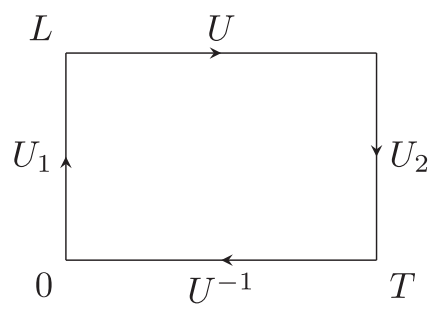

FIG. 2. Holonomy around the boundary of the plaquette.

Now we should be more accurate with the definition of the holonomy around the boundary (see Fig. 2). For each $U_{i}$, we have

$$
\begin{gathered}
U_{1}=\operatorname{Pexp}\left(\int_{0}^{L} A_{1}(x, 0) \mathrm{d} x\right), \\
U=\operatorname{Pexp}\left(\int_{0}^{T} A_{1}(L, t) \mathrm{d} t\right), \\
U_{2}=\operatorname{Pexp}\left(\int_{0}^{1} A_{1}(x, T) \frac{\mathrm{d} x}{\mathrm{~d} \sigma_{2}} \mathrm{~d} \sigma_{2}\right) \\
\text { with } \quad x\left(\sigma_{2}=0\right)=L, x\left(\sigma_{2}=1\right)=0 .
\end{gathered}
$$

Since we have two kinds of vector potentials defined by the boundary conditions with $\Omega_{0}(0)=\Omega_{0}(L)$ and $\Omega_{1}(0)=\Omega_{1}(L) z_{1}, \quad z_{1} \neq \mathrm{Id}$, the total partition function can be represented as the following sum:

$$
Z=\frac{1}{2}\left(Z_{0}+Z_{1}\right)
$$

where the factor $1 / 2$ comes from the normalization of the Haar measure to give volume one. Now $Z_{0}$ corresponds to the periodic boundary conditions, as in the case of pure $S U(2)$, and we already know the answer:

$$
Z_{0}=\sum_{m=0}^{\infty} \mathrm{e}^{-a m(m+2) / 4}
$$

To compute $Z_{1}$, we use the boundary conditions to derive

$$
\begin{aligned}
U_{2} & =\Omega_{1}(0) \operatorname{Pexp}\left(\int_{0}^{1} A_{1}(x, 0) \frac{\mathrm{d} x}{\mathrm{~d} \sigma_{2}} \mathrm{~d} \sigma_{2}\right) \Omega_{1}^{-1}(L) \\
& =\Omega_{1}(0) U_{1}^{-1} \Omega_{1}^{-1}(L) .
\end{aligned}
$$

Then the partition function for the cylinder is

$$
Z_{1}\left(a, U_{1}, U_{2}\right)=\sum_{R} \mathrm{e}^{-a C_{2}(R)} \chi_{R}\left(U_{1}\right) \chi_{R}\left(U_{2}\right)
$$

Applying the gluing procedure and integrating over $U_{1}$, we arrive at

$$
\begin{aligned}
Z_{1} & =\sum_{R} \mathrm{e}^{-a C_{2}(R)} \int \mathrm{d} U_{1} \chi_{R}\left(U_{1}\right) \chi_{R}\left(z_{1} U_{1}^{-1}\right) \\
& =\sum_{R} \mathrm{e}^{-a C_{2}(R)} \frac{\chi_{R}\left(z_{1}\right)}{\operatorname{dim} R}
\end{aligned}
$$

Using the Weyl character formula for the $S U(2)$ case

$\chi_{R}\left(\left(\begin{array}{cc}\mathrm{e}^{\imath \phi} & 0 \\ 0 & \mathrm{e}^{-\imath \phi}\end{array}\right)\right)=\frac{\sin (n \phi)}{\sin (\phi)}, \quad n=\operatorname{dim} R$,

we get $\chi_{R}\left(z_{1}\right)=n(-1)^{n+1}$ and

$$
Z_{1}=\sum_{m=0}^{\infty}(-1)^{m} \mathrm{e}^{-a m(m+2) / 4}
$$

Thus, the answer for the total partition function is

$$
Z=\sum_{k=0}^{\infty} \mathrm{e}^{-a k(k+1)}
$$

which coincides with the general answer (2.4) for the group $S O(3)$.

\section{A. Adding $w_{2}$ to $S U(2) / \mathbb{Z}_{2}$}

Following [40,51], we use a topological approach to the calculation of the partition function with the second StiefelWhitney class $w_{2}$ added to the Lagrangian:

$$
Z^{\mathrm{SW}}=\int \mathcal{D} A \mathrm{e}^{l S_{\mathrm{YM}}+l \theta w_{2}},
$$

where the dependence on $\theta$ is $2 \pi$-periodic and different possible values of theta in the $S U(2)$ case are $\theta=0, \pi$. Since $w_{2}$ only depends on the topological type of the bundle, the path integral splits into two parts, corresponding to the trivial and nontrivial $S O(3)$-bundles over the torus. The trivial bundle is defined by the boundary conditions (3.4) with $\Omega_{0}(0)=\Omega_{0}(L)$, and the value of $w_{2}$ is 0 . The nontrivial bundle is defined by the boundary conditions (3.4) with $\Omega_{1}(0)=\Omega_{1}(L) z_{1}$, and the value of $w_{2}$ is 1 . In this way we get the following answer:

$Z^{\mathrm{SW}}(\theta=\pi)=\frac{1}{2}\left(Z_{0}+\mathrm{e}^{\imath \pi} Z_{1}\right)=\sum_{k=0}^{\infty} \mathrm{e}^{-a(2 k+1)(2 k+3) / 4}$,

where SW stands for Stiefel-Whitney.

\section{IV. $S U(N) / \mathbb{Z}_{N}$ GAUGE THEORY}

For the group $S U(N) / \mathbb{Z}_{N}$, we have $N$ nonequivalent periodic boundary conditions (3.4) with 
$\Omega_{k}(0)=\Omega_{k}(L) z_{k}, \quad z_{k} \in \mathbb{Z}_{N}, \quad k=0,1, \ldots, N-1$.

Repeating the steps from the previous section, we write the partition function as

$$
Z=\frac{1}{N} \sum_{k=0}^{N-1} Z_{k}
$$

where

$$
Z_{k}=\sum_{R} \mathrm{e}^{-a C_{2}(R)} \frac{\chi_{R}\left(z_{k}\right)}{\operatorname{dim} R}
$$

We can add the $\theta$ term to the Lagrangian as in (3.17) with $w_{2}$ replaced by an invariant of $P S U(N)$ bundles $u_{2} \in$ $H^{2}\left(\mathbf{T}^{2} ; \mathbb{Z}_{N}\right)$ [18]. Allowing theta to take more values inside the $[0,2 \pi)$ interval and labeling these values by $\kappa$, we get

$$
\theta_{\kappa}=\frac{2 \pi \kappa}{N}, \quad \kappa=0, \ldots, N-1 .
$$

Thus, each $Z_{k}$ acquires the factor of $\mathrm{e}^{\imath \theta_{k} k}$, and the corresponding partition function is

$$
Z_{\kappa}^{\mathrm{SW}} \equiv Z^{\mathrm{SW}}\left(\theta=\theta_{\kappa}\right)=\frac{1}{N} \sum_{k=0}^{N-1} \mathrm{e}^{2 \pi \imath \kappa k / N} Z_{k} .
$$

\section{A. Example: $S U(3) / \mathbb{Z}_{3}$}

Irreducible representations of $S U(3)$ can be labeled by the Dynkin coefficients $(n, m)$. The two fundamental weights of $S U(3)$ are

$$
\mu^{1}=\left(\frac{1}{2}, \frac{1}{2 \sqrt{3}}\right), \quad \mu^{2}=\left(0, \frac{1}{\sqrt{3}}\right) .
$$

For the characters of $z_{k}$ in the representation $(n, m)$, this gives

$\chi_{(n, m)}\left(z_{k}\right)=\operatorname{dim} R_{(n, m)} \mathrm{e}^{2 \pi i k(n+2 m) / 3}, \quad k=0,1,2$.

Since

$$
\sum_{k=0}^{2} \mathrm{e}^{2 \pi i k(n+2 m) / 3}=3 \delta([n+2 m] \bmod 3), \quad \delta(n) \equiv \delta_{n, 0}
$$

and

$$
C_{2}\left(R_{(n, m)}\right)=\left(n^{2}+m^{2}+n m+3 n+3 m\right) / 3,
$$

we derive for the partition function

$Z=\sum_{n, m=0}^{\infty} \mathrm{e}^{-a\left(n^{2}+m^{2}+n m+3 n+3 m\right) / 3} \delta([n+2 m] \bmod 3)$,

where due to the Kronecker delta function the only nonzero terms are those that have $n+2 m \equiv 0(\bmod 3)$.

Adding $u_{2}$ with $\theta=\theta_{\kappa}$ changes the argument of the delta function by $\kappa$, and we get

$$
Z_{\kappa}^{\mathrm{SW}}=\sum_{n, m=0}^{\infty} \mathrm{e}^{-a\left(n^{2}+m^{2}+n m+3 n+3 m\right) / 3} \delta([\kappa+n+2 m] \bmod 3) .
$$

\section{B. General case: $S U(N) / \mathbb{Z}_{N}$}

Labeling representations of $S U(N)$ by the Dynkin coefficients $\left(q_{1}, \ldots, q_{N-1}\right) \equiv \boldsymbol{q}$ and using the fundamental weights, we derive for the characters of $z_{k}$ in the representation $\left(q_{1}, \ldots, q_{N-1}\right)$,

$$
\begin{aligned}
\chi_{\boldsymbol{q}}\left(z_{k}\right) & =\operatorname{dim} R_{q} \mathrm{e}^{2 \pi l k\left(q_{1}+2 q_{2}+\cdots+(N-1) q_{N-1}\right) / N}, \\
k & =0,1, \ldots, N-1 .
\end{aligned}
$$

Then with the help of the simple identity

$$
\sum_{k=0}^{N-1} \mathrm{e}^{2 \pi k k n / N}=N \delta(n \bmod N),
$$

we get for the partition function

$$
Z=\sum_{q_{1}, \ldots, q_{N-1}=0}^{\infty} \mathrm{e}^{-a C_{2}\left(R_{q}\right)} \delta\left(\left[\sum_{j=1}^{N-1} j q_{j}\right] \bmod N\right),
$$

where the only nonzero terms are those that have $\sum_{j=1}^{N-1} j q_{j} \equiv 0(\bmod N)$. The eigenvalues of the quadratic Casimir operator in (4.14) are given by [52]

$$
C_{2}\left(R_{\boldsymbol{q}}\right)=\sum_{j, k=1}^{N-1} q_{j}\left(q_{k}+2\right) G^{k j},
$$

where $G^{i j}$ is the inverse of the symmetrized Cartan matrix $G_{i j}[53]$,

$$
G_{i j} \equiv \frac{8\left(\alpha_{i}, \alpha_{j}\right)}{\left(\alpha_{i}, \alpha_{i}\right)\left(\alpha_{j}, \alpha_{j}\right)}
$$

and we are using the normalization, which provides the Killing metric of the form $g_{a b}=\frac{1}{2} \delta_{a b}$ and $\left(\alpha_{i}, \alpha_{i}\right)=2$. 
Adding the usual $\theta$ term with $\theta=\theta_{\kappa}$, we obtain

$$
\begin{aligned}
Z_{\kappa}^{\mathrm{SW}} & =\sum_{q_{1}, \ldots, q_{N-1}=0}^{\infty} \mathrm{e}^{-a C_{2}\left(R_{q}\right)} \delta\left(\left[\kappa+\sum_{j=1}^{N-1} j q_{j}\right] \bmod N\right), \\
\kappa & =1, \ldots, N-1 .
\end{aligned}
$$

\section{LOOKING FOR TWO VACUA IN $S U(N) / \Gamma$, $\Gamma \subset \mathbb{Z}_{N}$ GAUGE THEORY}

In this section we will consider the more general case when the factor group is taken with respect to the subgroup $\Gamma$ of the center of $S U(N)$. By this point, we have gone through several derivations of the partition functions, and it is clear what the generalization is of (4.17) for $\Gamma \neq \mathbb{Z}_{N}$. If the order of $\Gamma$ is $n$, then we have $n$ nonequivalent periodic boundary conditions (3.4), and the corresponding partition function is

$$
\begin{aligned}
Z_{\kappa}^{\mathrm{SW}} & =\sum_{q_{1}, \ldots, q_{N-1}=0}^{\infty} \mathrm{e}^{-a C_{2}\left(R_{q}\right)} \delta\left(\left[\kappa+\sum_{j=1}^{N-1} j q_{j}\right] \bmod n\right), \\
\kappa & =1, \ldots, n-1 .
\end{aligned}
$$

\section{A. $N=4$}

We start with the explicit answer for the case of $S U(4) / \mathbb{Z}_{4}$ :

$$
\begin{aligned}
\left.Z_{\kappa}^{\mathrm{SW}}\right|_{\Gamma=\mathbb{Z}_{4}}= & \sum_{q_{1}, q_{2}, q_{3}=0}^{\infty} \mathrm{e}^{-a C_{2}\left(R_{\left(q_{1}, q_{2}, q_{3}\right)}\right)} \\
& \times \delta\left(\left[\kappa+\sum_{j=1}^{3} j q_{j}\right] \bmod 4\right), \quad \kappa=1, \ldots, 3,
\end{aligned}
$$

where

$$
\begin{aligned}
C_{2}\left(R_{\left(q_{1}, q_{2}, q_{3}\right)}\right)= & \frac{1}{8}\left(3 q_{1}^{2}+4 q_{2}^{2}+3 q_{3}^{2}+4 q_{1} q_{2}\right. \\
& \left.+2 q_{1} q_{3}+4 q_{2} q_{3}+12 q_{1}+16 q_{2}+12 q_{3}\right) .
\end{aligned}
$$

As it can be checked directly, there is no such value of $\kappa$ that would produce two vacua. However, we can also consider the case of $S U(4) / \mathbb{Z}_{2}$ with $\kappa=1$ and the following partition function:

$$
\begin{aligned}
\left.Z_{1}^{\mathrm{SW}}\right|_{\Gamma=\mathbb{Z}_{2}}= & \sum_{q_{1}, q_{2}, q_{3}=0}^{\infty} \mathrm{e}^{-a C_{2}\left(R_{\left(q_{1}, q_{2}, q_{3}\right)}\right)} \\
& \delta\left(\left[1+\sum_{j=1}^{3} j q_{j}\right] \bmod 2\right) .
\end{aligned}
$$

In this case the two vacua contributions are given by $\boldsymbol{q}=$ $(1,0,0)$ and $\boldsymbol{q}=(0,0,1)$.

\section{B. General case of $S U(2 N) / \mathbb{Z}_{2}$ with $N>1$}

It is easy to show that the first nontrivial example of $2 \mathrm{D}$ theory with two vacua discussed earlier is just one of the infinite series of $S U(2 N) / \mathbb{Z}_{2}$ theories with $N>1$. We again consider the partition function $\left.Z_{\kappa}^{\mathrm{SW}}\right|_{\Gamma=\mathbb{Z}_{2}}$ with $\kappa=1$ or, equivalently, $\theta=\pi$ :

$$
\left.Z_{1}^{\mathrm{SW}}\right|_{\Gamma=\mathbb{Z}_{2}}=\sum_{q_{1}, \ldots, q_{2 N-1}=0}^{\infty} \mathrm{e}^{-a C_{2}\left(R_{q}\right)} \delta\left(\left[1+\sum_{j=1}^{2 N-1} j q_{j}\right] \bmod 2\right) .
$$

To show that these theories have two vacua, we need the following facts about the inverse Cartan matrix $G^{i j}$. The first fact is that all elements of this matrix are strictly positive:

$$
\forall i, j: G^{i j}>0 .
$$

Second, the diagonal elements $G^{i i}$ are given by $[53,54]$

$$
G^{i i}=\frac{i(2 N-i)}{4 N}, \quad i \leq 2 N-1 .
$$

And finally, the following relations hold:

$$
\begin{gathered}
\forall j=2, \ldots, N: \sum_{i=1}^{2 N-1} G^{i 1}<\sum_{i=1}^{2 N-1} G^{i j}, \\
\forall j=1, \ldots, N-1: \sum_{i=1}^{2 N-1} G^{i, N-j}=\sum_{i=1}^{2 N-1} G^{i, N+j} .
\end{gathered}
$$

Thus, the two vacua contributions are coming from

$$
\boldsymbol{q}=(1,0, \ldots, 0) \quad \text { and } \quad \boldsymbol{q}=(0, \ldots, 0,1) .
$$

Notice that due to the superselection rules

$$
\left\langle R_{2} \mid R_{1}\right\rangle=\int \mathrm{d} U \chi_{R_{1}}(U) \chi_{R_{2}}\left(U^{-1}\right)=\delta_{R_{1}, R_{2}}
$$

we indeed have two different ground states that indicate spontaneous symmetry breaking.

$$
\text { C. } \Gamma \neq \mathbb{Z}_{2}
$$

By studying some particular examples with low enough values of $N$, one can check that the following theories with $\Gamma \neq \mathbb{Z}_{2}$ have two vacuum states: $S U(8) / \mathbb{Z}_{4}$ with $\kappa=2, S U(12) / \mathbb{Z}_{4}$ with $\kappa=2, S U(12) / \mathbb{Z}_{6}$ with $\kappa=3$, $S U(16) / \mathbb{Z}_{4}$ with $\kappa=2$, and $S U(16) / \mathbb{Z}_{8}$ with $\kappa=4$. Basically, any $S U(2 N m) / \mathbb{Z}_{2 m}$ theory with $\kappa=m$ and 
$N>1, m>1$ is a candidate for having a twofold degenerate vacuum. The only obstacle to making this statement true, in general, is that for higher values of $N$ and $m$ there could be, in principle, states with energies lower than the energy of the following two states:

$$
\begin{gathered}
q_{m}=1, \quad \forall j \neq m: q_{j}=0 \quad \text { and } q_{(2 N-1) m}=1, \\
\forall j \neq(2 N-1) m: q_{j}=0 .
\end{gathered}
$$

However, explicit computations for a number of different values of $N$ and $m$ suggest that the above states are always the lowest energy states of the theory with $\kappa=m$. If we assume that there are states with even lower energies, then they will also come in pairs. This allows us to conclude that the vacuum of the $S U(2 \mathrm{Nm}) / \mathbb{Z}_{2 m}$ theory with $\kappa=m$ is at least twofold degenerate. Moreover, the lack of discrete symmetries with orders higher than 2 hints that the twofold degeneracy is the only option.

\section{SPONTANEOUS SYMMETRY BREAKING IN $S U(2 N) / \mathbb{Z}_{2}$ THEORIES WITH $\theta=\pi, N>1$}

If we look at the Dynkin coefficients corresponding to the two vacuum states, we see that these states are given by the fundamental and antifundamental representations of $S U(2 N)$. Hence, the question is what transformation brings us from one representation to its complex conjugate. Since the wave functions in the propagator (2.1) are given by

$$
\begin{aligned}
\langle U \mid R\rangle & =\chi_{R}(U)=\operatorname{Tr}_{R}(U), \\
U & =\operatorname{Pexp}\left(\int_{0}^{L} A_{1}(x, t) \mathrm{d} x\right),
\end{aligned}
$$

the transformation $A_{1} \rightarrow-A_{1}^{T}$ yields $U \rightarrow\left(U^{-1}\right)^{T}=U^{*}$ and $\chi_{R}(U) \rightarrow \chi_{R^{*}}(U)$. From the Gauss law constraint, one could derive the following transformation rules for the $\mathbf{C}$, $\mathbf{P}$, and $\mathbf{T}$ operators:

$$
\begin{array}{ll}
\mathbf{C}: e \rightarrow-e, & A_{1} \rightarrow-A_{1}^{T}, \\
\mathbf{P}: x \rightarrow-x, & A_{1} \rightarrow-A_{1}, \\
\mathbf{T}: t \rightarrow-t, & A_{1} \rightarrow-A_{1}^{T} .
\end{array}
$$

Thus, the $\mathbf{C}$-symmetry (as well as $\mathbf{T}$ ) is spontaneously broken, and the overall CPT-symmetry is conserved since both $\mathbf{C T}$ and $\mathbf{P}$ act trivially on the wave functions $\chi_{R}(U)$.

Spontaneously broken $\mathbf{C}$ - and $\mathbf{T}$-symmetries lead to the domain wall between the two vacuum states $\chi_{F}(U)$ and $\chi_{\bar{F}}(U)$. In the theories under consideration there is a discrete one-form symmetry $\mathbb{Z}_{N}$, generated by a local unitary operator $\mathcal{O}[8,55]$. This local operator picks up a phase when crossing the domain wall. To figure out the phase, we consider the $\mathbb{Z}_{2 N}$ subgroup before factoring out $\mathbb{Z}_{2}$. As before, the corresponding characters are given by

$$
\begin{aligned}
\chi_{q}\left(z_{k}\right) & =\operatorname{dim} R_{q} \mathrm{e}^{2 \pi k k\left(q_{1}+2 q_{2}+\cdots+(2 N-1) q_{2 N-1}\right) /(2 N)}, \\
k & =0,1, \ldots, 2 N-1 .
\end{aligned}
$$

After factoring out $\mathbb{Z}_{2}$, the generator of $\mathbb{Z}_{N}$ corresponds to $z_{1}$, and its action on the wave functions is simply

$$
\mathcal{O}|F\rangle=\mathrm{e}^{\pi \imath / N}|F\rangle, \quad \mathcal{O}|\bar{F}\rangle=\mathrm{e}^{-\pi \imath / N}|\bar{F}\rangle,
$$

where $\mathcal{O}^{N}=1$ due to the fact that $(-1) \in \mathbb{Z}_{2}$ in fundamental and antifundamental representations. Here, we also assume that adding a second Stiefel-Whitney class only affects the $\mathbb{Z}_{2}$-charges of the states and $\mathbb{Z}_{N}$-charges remain the same. In this way the relation between the two expectation values reads

$$
\langle\mathcal{O}\rangle_{F}=\mathrm{e}^{2 \pi l / N}\langle\mathcal{O}\rangle_{\bar{F}},
$$

which fixes the phase factor picked by $\mathcal{O}$ upon crossing the domain wall to be $\mathrm{e}^{2 \pi l / N}$. Then the action of charge conjugation on $\mathcal{O}$ can be inferred from

$\mathbf{C O} \mathbf{C}^{-1}|F\rangle=\mathrm{e}^{-\pi l / N}|F\rangle, \quad \mathbf{C O} \mathbf{C}^{-1}|\bar{F}\rangle=\mathrm{e}^{\pi \imath / N}|\bar{F}\rangle$.

The latter implies

$$
\mathbf{C O C}^{-1}=\mathcal{O}^{-1}
$$

\section{CONCLUSION}

In this paper we described a new mechanism of spontaneous symmetry breaking in pure two dimensional YangMills theories. Using the well-developed methods for computing the 2D partition functions on compact manifolds $[33,40,45]$ and 't Hooft's idea of twisted boundary conditions [50], we analyzed the wide range of systems and presented the corresponding partition functions. We observed that there is no spontaneous symmetry breaking in $P S U(N)$ theories for any $N$, which led us to consider a more general case of $S U(N) / \Gamma$ theories with $\Gamma$ being the subgroup of the center of $S U(N)$. Within this class of systems we found many examples of theories with a degenerate vacuum state, and in the particular case of $S U(2 N) / \mathbb{Z}_{2}, N>1$, we proved that spontaneous symmetry breaking occurs. Additionally, we argued that the corresponding order parameter is given by the generator $\mathcal{O}$ of the $\mathbb{Z}_{N}$ one-form symmetry. There are still a number of questions left to answer. In particular, it will be interesting to prove that the same mechanism of spontaneous symmetry breaking takes place in $S U(2 \mathrm{Nm}) / \mathbb{Z}_{2 m}$ theories. As we mentioned in the introduction, the spontaneous symmetry breaking could also imply the existence of the mixed anomaly, which may be a topic of a separate study. 
Another interesting question is how to perturb the theories under consideration so that the vacuum is no longer degenerate. As we discussed earlier, the two vacuum states are given by the fundamental and antifundamental representations. The reason is that the energy of the states is proportional to the eigenvalue of the quadratic Casimir operator, which does not distinguish between any given representation and its complex conjugate. However, if we find a way to modify the theory such that the Hamiltonian will include higher order Casimir operators, we will lift the degeneracy. Indeed, such modifications exist and were discussed in $[40,45]$. Below we will briefly repeat the arguments from $[40,45]$ and show how to perturb the Hamiltonian by the cubic Casimir operator.

In $2 \mathrm{D}$ it is possible to define the Lie algebra valued scalar $f=* F$. Thus, we can add an irrelevant operator $\operatorname{Tr}\left(f^{k}\right)$ with any $k>2$ as a perturbation to the original theory. This perturbation will affect the Hamiltonian by introducing new Casimirs of order less than or equal to $k$ [45]. Since our goal is to distinguish between the fundamental and antifundamental representations of $S U(N)$, it is enough to consider $k=3$. Then the Hamiltonian is a linear combination of the quadratic and cubic Casimirs (in the representation basis). For example, consider the case of the $S U(4) / \mathbb{Z}_{2}$ theory. Without the perturbation the energy is proportional to $C_{2}\left(R_{\boldsymbol{q}}\right)$ (5.3) and symmetric under the permutation of $q_{1}$ and $q_{3}$. In the perturbed system the energy acquires nonzero contributions proportional to

$C_{3}\left(R_{q}\right)=\frac{3}{16}\left(q_{1}-q_{3}\right)\left(q_{1}+q_{3}+2\right)\left(q_{1}+2 q_{2}+q_{3}+4\right)$,

which is clearly antisymmetric in $q_{1}$ and $q_{3}$. Hence, the energies of the two original vacuum states with $\boldsymbol{q}=$ $(1,0,0)$ and $\boldsymbol{q}=(0,0,1)$ will get different corrections, and the resulting system will have a single vacuum state. So far, we described one possible approach to lifting the degeneracy in the $S U(2 N) / \mathbb{Z}_{2}$ theories with $\theta=\pi$ and $N>1$. The existence of any other approaches and detailed calculations for the case of general $N$ are left for future work.

\section{ACKNOWLEDGMENTS}

G. A. would like to thank Zohar Komargodski for initiating the work and many valuable discussions and Konstantinos Roumpedakis for useful remarks and discussions. The work was supported by the Foundation for the Advancement of Theoretical Physics and Mathematics "BASIS" Grant No. 18-1-1-50-3 and in part by RFBR Grants No. 18-02-01081-A, No. 18-31-20046-mol_a_ved, No. 18-51-05015-Arm_a, and No. 19-51-18006-Bolg_a.
[1] J. Lowenstein and J. Swieca, Quantum electrodynamics in two dimensions, Ann. Phys. (N.Y.) 68, 172 (1971).

[2] G. 't Hooft, A planar diagram theory for strong interactions, Nucl. Phys. B72, 461 (1974).

[3] S. Coleman, More about the massive Schwinger model, Ann. Phys. (N.Y.) 101, 239 (1976).

[4] C. G. Callan, R. F. Dashen, and D. J. Gross, The structure of the gauge theory vacuum, Phys. Lett. B 63, 334 (1976).

[5] A. M. Polyakov, Quark confinement and topology of gauge theories, Nucl. Phys. B120, 429 (1977).

[6] E. Witten, Theta-vacua in two-dimensional quantum chromodynamics, Il Nuovo Cimento A 51, 325 (1979).

[7] A. Kapustin and N. Seiberg, Coupling a QFT to a TQFT and duality, J. High Energy Phys. 04 (2014) 001.

[8] D. Gaiotto, A. Kapustin, N. Seiberg, and B. Willett, Generalized global symmetries, J. High Energy Phys. 02 (2015) 172.

[9] G. 't Hooft, Naturalness, chiral symmetry, and spontaneous chiral symmetry breaking, NATO ASI Ser., Ser. B 59, 135 (1980).

[10] A. N. Redlich, Parity violation and gauge noninvariance of the effective gauge field action in three dimensions, Phys. Rev. D 29, 2366 (1984).
[11] A. N. Redlich, Gauge Noninvariance and Parity Nonconservation of Three-Dimensional Fermions, Phys. Rev. Lett. 52, 18 (1984).

[12] A. Kapustin and R. Thorngren, Anomalous Discrete Symmetries in Three Dimensions and Group Cohomology, Phys. Rev. Lett. 112, 231602 (2014).

[13] A. Kapustin and R. Thorngren, Anomalies of discrete symmetries in various dimensions and group cohomology, arXiv:1404.3230.

[14] N. Seiberg and E. Witten, Gapped boundary phases of topological insulators via weak coupling, Prog. Theor. Exp. Phys. 2016, 12C101 (2016).

[15] F. Benini, P.-S. Hsin, and N. Seiberg, Comments on global symmetries, anomalies, and duality in $(2+1) d$, J. High Energy Phys. 04 (2017) 135.

[16] D. Gaiotto, A. Kapustin, Z. Komargodski, and N. Seiberg, Theta, time reversal and temperature, J. High Energy Phys. 05 (2017) 091.

[17] Y. Tanizaki, T. Misumi, and N. Sakai, Circle compactification and 't Hooft anomaly, J. High Energy Phys. 12 (2017) 056.

[18] Z. Komargodski, A. Sharon, R. Thorngren, and X. Zhou, Comments on Abelian Higgs models and persistent order, SciPost Phys. 6, 3 (2019). 
[19] Z. Komargodski, T. Sulejmanpasic, and M. Unsal, Walls, anomalies, and deconfinement in quantum antiferromagnets, Phys. Rev. B 97, 054418 (2018).

[20] Y. Tanizaki, Y. Kikuchi, T. Misumi, and N. Sakai, Anomaly matching for the phase diagram of massless $\mathbb{Z}_{N}$-QCD, Phys. Rev. D 97, 054012 (2018).

[21] T. Misumi, Y. Tanizaki, and M. Unsal, Fractional $\theta$ angle, 't Hooft anomaly, and quantum instantons in charge-q multiflavor Schwinger model, J. High Energy Phys. 07 (2019) 018.

[22] C. Cordova, D. S. Freed, H. Tat Lam, and N. Seiberg, Anomalies in the space of coupling constants and their dynamical applications I, SciPost Phys. 8, 001 (2020).

[23] A. Cherman, T. Jacobson, Y. Tanizaki, and M. Unsal, Anomalies, a mod 2 index, and dynamics of $2 \mathrm{~d}$ adjoint QCD, SciPost Phys. 8, 072 (2020).

[24] C. Cordova and K. Ohmori, Anomaly obstructions to symmetry preserving gapped phases, arXiv:1910.04962.

[25] D. Kapec, R. Mahajan, and D. Stanford, Matrix ensembles with global symmetries and 't Hooft anomalies from $2 \mathrm{~d}$ gauge theory, J. High Energy Phys. 04 (2020) 186.

[26] Z. Wan, J. Wang, and Y. Zheng, Quantum 4d Yang-Mills theory and time-reversal symmetric 5d higher-gauge topological field theory, Phys. Rev. D 100, 085012 (2019).

[27] Z. Wan, J. Wang, and Y. Zheng, New higher anomalies, SU(N) Yang-Mills gauge theory and $\mathbb{C P}^{N-1}$ sigma model, Ann. Phys. (Amsterdam) 414, 168074 (2020).

[28] I. Affleck, Nonlinear $\sigma$ Model at $\theta=\pi$ : Euclidean Lattice Formulation and Solid-On-Solid Models, Phys. Rev. Lett. 66, 2429 (1991).

[29] E. Sharpe, Undoing decomposition, Int. J. Mod. Phys. A 34, 1950233 (2019).

[30] T. Pantev and E. Sharpe, Notes on gauging noneffective group actions, arXiv:hep-th/0502027.

[31] M. Ando, S. Hellerman, A. Henriques, T. Pantev, and E. Sharpe, Cluster decomposition, T-duality, and gerby CFT's, Adv. Theor. Math. Phys. 11, 751 (2007).

[32] E. Sharpe, Decomposition in diverse dimensions, Phys. Rev. D 90, 025030 (2014).

[33] A. Migdal, Recursion relations in gauge theories, Zh. Eksp. Teor. Fiz. 69, 810 (1975) [Sov. Phys. JETP 42, 413 (1976)], https://ui.adsabs.harvard.edu/abs/1976JETP...42..413M/ abstract.

[34] V. A. Kazakov and I. K. Kostov, Non-linear strings in twodimensional $U(\infty)$ gauge theory, Nucl. Phys. B176, 199 (1980).

[35] V. A. Kazakov, Wilson loop average for an arbitrary contour in two-dimensional U(N) gauge theory, Nucl. Phys. B179, 283 (1981).

[36] M. F. Atiyah and R. Bott, The Yang-Mills equations over Riemann surfaces, Phil. Trans. R. Soc. A 308, 523 (1983).
[37] K. H. O'Brien and J.-B. Zuber, Strong coupling expansion of large-N QCD and surfaces, Nucl. Phys. B253, 621 (1985).

[38] D. S. Fine, Quantum Yang-Mills on the two-sphere, Commun. Math. Phys. 134, 273 (1990).

[39] D. S. Fine, Quantum Yang-Mills on a Riemann surface, Commun. Math. Phys. 140, 321 (1991).

[40] E. Witten, On quantum gauge theories in two dimensions, Commun. Math. Phys. 141, 153 (1991).

[41] D. J. Gross and W. Taylor, Two-dimensional QCD is a string theory, Nucl. Phys. B400, 181 (1993).

[42] I. K. Kostov, U(N) gauge theory and lattice strings, Nucl. Phys. B415, 29 (1994).

[43] J. Minahan and A. Polychronakos, Classical solutions for two-dimensional QCD on the sphere, Nucl. Phys. B422, 172 (1994).

[44] S. Cordes, G. Moore, and S. Ramgoolam, Large N 2D YangMills theory and topological string theory, Commun. Math. Phys. 185, 543 (1997).

[45] S. Cordes, G. Moore, and S. Ramgoolam, Lectures on 2D Yang-Mills theory, equivariant cohomology and topological field theories, Nucl. Phys. B, Proc. Suppl. 41, 184 (1995).

[46] V. Kazakov, String Theory, Gauge Theory and Quantum Gravity '93, in Proceedings of the Trieste Spring School and Workshop, ICTP, Trieste, Italy, 1993 (World Scientific, Singapore, 1994), p. 29-56, https://doi.org/10.1142/ 9789814447072_0002.

[47] M. Blau and G. Thompson, Quantum Maxwell theory on arbitrary surfaces (1991), https://inis.iaea.org/collection/ NCLCollectionStore/_Public/22/061/22061437.pdf?r=1.

[48] M. Blau and G. Thompson, Quantum Yang-Mills theory on arbitrary surfaces, Int. J. Mod. Phys. A 07, 3781 (1992).

[49] M. Blau and G. Thompson, Lectures on 2d gauge theories: Topological aspects and path integral techniques, arXiv: hep-th/9310144.

[50] G. 't Hooft, A property of electric and magnetic flux in nonabelian gauge theories, Nucl. Phys. B153, 141 (1979).

[51] A. Alekseev, O. Chekeres, and P. Mnev, Wilson surface observables from equivariant cohomology, J. High Energy Phys. 11 (2015) 093.

[52] P. Di Francesco, P. Mathieu, and D. Senechal, Conformal Field Theory (Springer-Verlag, New York, 1997), https:// doi.org/10.1007/978-1-4612-2256-9.

[53] J. Fuchs and C. Schweigert, Symmetries, Lie Algebras and Representations (Cambridge University Press, Cambridge, England, 1997).

[54] R. Slansky, Group theory for unified model building, Phys. Rep. 79, 1 (1981).

[55] L. P. Kadanoff and H. Ceva, Determination of an operator algebra for the two-dimensional Ising model, Phys. Rev. B 3, 3918 (1971). 